\title{
Blockchain for federated learning toward secure distributed machine learning systems: a systemic survey
}

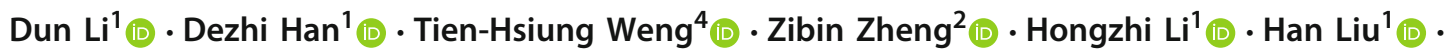

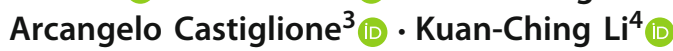

Accepted: 24 October 2021 / Published online: 20 November 2021

(c) The Author(s), under exclusive licence to Springer-Verlag GmbH Germany, part of Springer Nature 2021

\begin{abstract}
Federated learning $(F L)$ is a promising decentralized deep learning technology, which allows users to update models cooperatively without sharing their data. $F L$ is reshaping existing industry paradigms for mathematical modeling and analysis, enabling an increasing number of industries to build privacy-preserving, secure distributed machine learning models. However, the inherent characteristics of $F L$ have led to problems such as privacy protection, communication cost, systems heterogeneity, and unreliability model upload in actual operation. Interestingly, the integration with Blockchain technology provides an opportunity to further improve the $F L$ security and performance, besides increasing its scope of applications. Therefore, we denote this integration of Blockchain and $F L$ as the Blockchain-based federated learning $(B C F L)$ framework. This paper introduces an in-depth survey of $B C F L$ and discusses the insights of such a new paradigm. In particular, we first briefly introduce the $F L$ technology and discuss the challenges faced by such technology. Then, we summarize the Blockchain ecosystem. Next, we highlight the structural design and platform of $B C F L$. Furthermore, we present the attempts ins improving $F L$ performance with Blockchain and several combined applications of incentive mechanisms in $F L$. Finally, we summarize the industrial application scenarios of $B C F L$.
\end{abstract}

Keywords Blockchain $\cdot$ Federated learning $\cdot$ Smart Contract $\cdot$ Incentive mechanism $\cdot$ Industrial Applications

Kuan-Ching Li

kuancli@pu.edu.tw

Dun Li

lidunshmtu@outlook.com

Dezhi Han

dzhan@shmtu.edu.cn

Tien-Hsiung Weng

thweng@pu.edu.tw

Zibin Zheng

zhzibin@mail.sysu.edu.cn

Arcangelo Castiglione

arcastiglione@unisa.it

1 College of Information Engineering at Shanghai Maritime University, Pudong, China

2 School of Software Engineering, Sun Yat-Sen University, Zhuhai, China

3 Dipartimento di Informatica, University of Salerno, Salerno, Italy

4 Department of Computer Science and Information Engineering, Providence University, Taichung City, Taiwan

\section{Introduction}

The quality and security of data are the keys to the development of machine learning and artificial intelligence $(A I)$. However, rich data is often privacy sensitive and large scale, which will hinder traditional methods to log into a data center and train there. Besides, most of the data and resources needed for effective training of machine learning models are owned by a few large technology companies, which is detrimental to privacy protection and further leads to centralization problems. Thus, a novel, distributed learning approach that allows large-scale joint modeling without publishing raw data becomes imperative. In this context, Federated learning $(F L)$ proposed by Google (Konečnỳ et al. 2016; Aledhari et al. 2020; Konečnỳ et al. 2016; McMahan et al. 2017) has recently received great attention at both the research and application levels.

Specifically, $F L$ is an emerging machine learning technology consisting of many mobile devices and a central storage server. This technology allows distributed model training using local datasets from large-scale nodes, such as mobile 
devices. $F L$ updates the parameters without uploading the original training data and then builds a shared model by aggregating the locally computed updates (Xu et al. 2020). A typical example is the FedAVG algorithm, which is based on the iterative model averaging proposed in McMahan et al. (2017). This method is robust and allows to generate imbalanced and independent, and constant distribution nonIID data distributions. The basic design structure of $F L$ is shown in Fig. 1. Based on this, $F L$ offers promising privacy protection for mobile devices while ensuring high learning performance.

However, despite the many benefits mentioned above, $F L$ still faces serious challenges. The gradient aggregation mechanism used for $F L$ makes the entire algorithmic model dependent on the control of a central node. So we need to address two trust issues: one is to ensure that there is a central node that all participants trust, and the other is to ensure that information about the operations of the central node is transparent. First of all, $F L$ relies on centralized databases and remains at risk of distributed denial of service $D D o S$ attacks and privacy breaches. Again, currently, $F L$ systems do not have suitable and transparent contribution evaluation mechanisms and incentive mechanisms to ensure continuous active training of training nodes. Finally, an effective distributed system needs to identify and prevent malicious nodes. However, the current $F L$ system does not provide adequate mechanisms to implement these operations.

Interestingly, Blockchain technology provides an opportunity to address the above challenges of $F L$. More precisely, through the combination of chain structure, tree structure, and graph structure, the Blockchain ensures secure storage and data traceability (Liang et al. 2020). Besides, through the consensus mechanism of proof-of-work $(P O W)$, Blockchain realizes the untamperability of data. In more detail, due to the validation process of Blockchain local training results, the proposed $B C F L$ framework can avoid the single point of failure (SPOF) and extend its federation scope to untrusted users in the public network. In addition, by providing rewards proportional to the size of the training samples, $B C F L$ can realize effective incentives and thus facilitate the union of more devices with a large number of training samples. Therefore, the Blockchain can be seen as a perfect complement for $F L$, providing it with improved interoperability, privacy, security, reliability, and scalability (Liang et al. 2021).

Although many papers involve different aspects of the $B C F L$ paradigm, there is no systematic investigation on this paradigm. In this article, we present a survey on a new paradigm for integrating Blockchain and $F L$. This survey denotes such a synthesis of Blockchain and $F L$ as Blockchain-based federated learning (BCFL) framework. To present a complete picture of $B C F L$-related studies, we surveyed the related works focusing on structure design, performance enhancement attempts, incentive mechanism

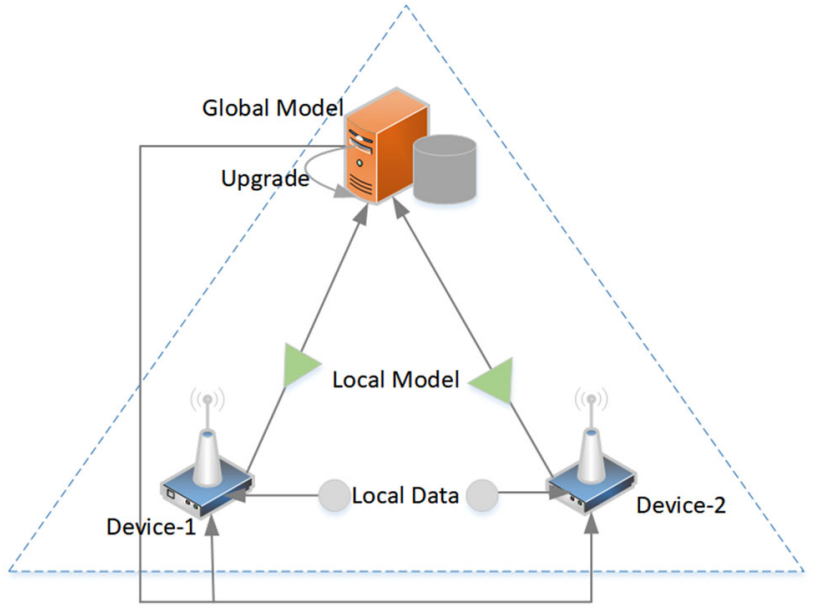

Fig. 1 The architecture of $F L$

design, and industrial applications of $B C F L$, in a period ranging from 2016 to 2021. Given the previous work, we aim to (i) provide a conceptual introduction to $F L$ and Blockchain technology, (ii) provide a systematic analysis of the potential of incorporating Blockchain into $F L$, and (iii) discuss the specific applications of $B C F L$ in depth.

In detail, the main contributions of this paper are summarized as follows.

- We provide an overview of the definition, architectural design, and deployed platform for Blockchain and $F L$ convergence.

- We provide a systematic survey on the studies dedicated to improving the performance of $F L$ by integrating block $F L$ systems.

- We survey the existing studies on effective incentive mechanisms for training nodes using Blockchain.

- We summarize the current feasible applications for $B C F L$ in industrial applications.

The rest of this article is organized as follows. We first introduce the related work in Sect. 2. Section 3 then introduces the background and fundamentals of $F L$ and Blockchain. Subsequently, Sect. 4 presents the convergence architecture and deployment platform of BCFL. Section 5 then summarizes the attempts to make appropriate improvements to the BCFL. Section 6 discusses the transparent contribution recognition and effective reward for clients in $B C F L$. Section 7 next summarizes the feasible application of $B C F L$. Finally, Sect. 8 concludes the paper. 


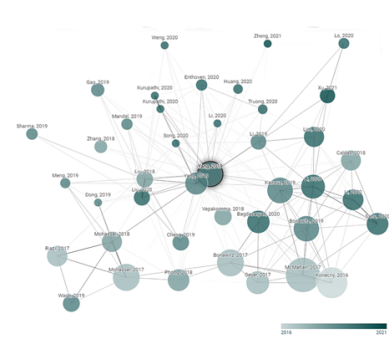

(a) Association of Yang's work (b) Association of Zheng's work

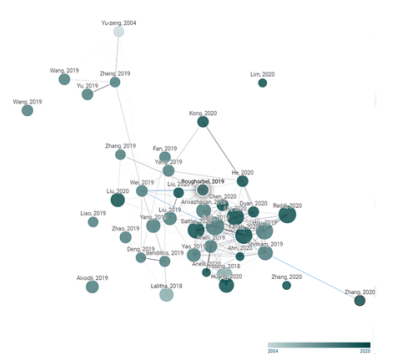

Fig. 2 The loosely related researches of Yang's work and Zheng's work

\section{Related work}

Currently, many studies have investigated the ideology, structure, and related research of FL and Blockchain, respectively. Particular, the works proposed in Konečnỳ et al. (2016), Konečnỳ et al. (2016), McMahan et al. (2017), Kairouz et al. (2019), Yang et al. (2019), Bonawitz et al. (2019), Yu et al. (2021), Li et al. (2020), Gu et al. (2019), Li et al. (2019), Mothukuri et al. (2021), Liang et al. (XXXX) comprehensively introduced the relevant information of $F L$, while the works proposed in Zheng et al. (2017), Kumar and Jaiswal (2019), Xiao et al. (2020), Gramoli (2020), Liang et al. (2020), Zhou et al. (2020), Saleh (2020), Li et al. (2020), Hewa et al. (2021), Liang et al. (2019), Xiao et al. (2020) summarized the main information concerning the structure and characteristics of Blockchain. In this work, we take Yang's work (Yang et al. 2019) and Zheng's work (Zheng et al. 2017) as baselines and organize the closely related research. As Fig. 2 shows, Yang's work is associated with more highly cited articles, and Zheng's work links more paper groups.

In conclusion, the technological development of $F L$ has attracted much attention, and the related research has shown an explosive growth trend. However, as Table 1 shows, there is no existing survey related to the combination of Blockchain and $F L$ in the literature. To fill this gap, we propose in this work the first survey that performs a thorough investigation of the relevant studies published in recent years on BCFL. Again, we systematically present the structural designs, deployed platforms, performance improvement, node incentive mechanisms, and the industrial applications of $B C F L$. Finally, based on the related works, Table 2 defines a list of acronyms and the definitions used in this survey.

\section{Background}

In this section, we provide all the background necessary to understand better and follow this paper. More precisely, we briefly introduce $F L$ integration in Sect. 3.1 and present Blockchain ecosystem in Section 3.2.

\subsection{Federated learning integration}

$F L$ refers to the calculation process that enables the data owner $F_{i}$ to perform model training and obtain the model $M_{F E D}$ without giving their data $D_{i}$ while ensuring that the gap between the effect $V_{F E D}$ of the model $M_{F E D}$ and the effect $V_{S U M}$ of the model $M_{S U M}$ is small enough. This calculation can be expressed as follows.

$$
\begin{aligned}
\omega_{t}^{i} & =\& \arg \min _{\omega_{t}^{i}} F\left(\omega_{t}^{i}\right) \\
F\left(\omega_{t}^{i}\right) \& & =\frac{1}{\left|\mathcal{D}_{i}\right|} \sum_{j \in \mathcal{D}_{i}} f_{j}\left(\omega_{t}^{i}\right)
\end{aligned}
$$

Where $\left|\mathcal{D}_{i}\right|$ is an arbitrarily small positive value, $1 \leq i \leq$ $n$, and $n$ is the number of participants to the system.

\subsubsection{Taxonomy of $F L$}

The basis of $F L$ is the data matrix. As shown in Fig. 3, based on the different distribution patterns of sample space and feature space of data, $F L$ can be divided into three categories: horizontal federated learning $(H F L)$, vertical federated learning $(V F L)$, and federated transfer learning (FTL) which divide the dataset horizontally (i.e., user dimension), longitudinal (i.e., feature dimensions), and non-dimensionally, respectively.

\subsubsection{The workflow of $F L$}

$F L$ systems generally consist of data holders and central servers. The amount of local data or the number of features of each data holder may not be enough to support successful model training. Therefore, support from other data holders is required. Figure 4 illustrates the $F L$ process for the clientserver architecture.

In a typical cooperative modeling process of $F L$, the training of local data by the data holders occurs only locally to protect data privacy. Next, the gradients generated by the iterations are used as interaction information after desensitization and uploaded to a third-party trusted server instead of local data, waiting for the server to return the aggregated parameters to update the model. In detail, the steps of $F L$ can be summarized as follows.

- Step 1. System Initialization. First, the central server sends the modeling task and seeks to participate in the client.

- Step 2. Local Calculation. After the joint modeling task is opened and the system parameters are initialized, each data holder will be required to perform local calculations according to the data locally first. 


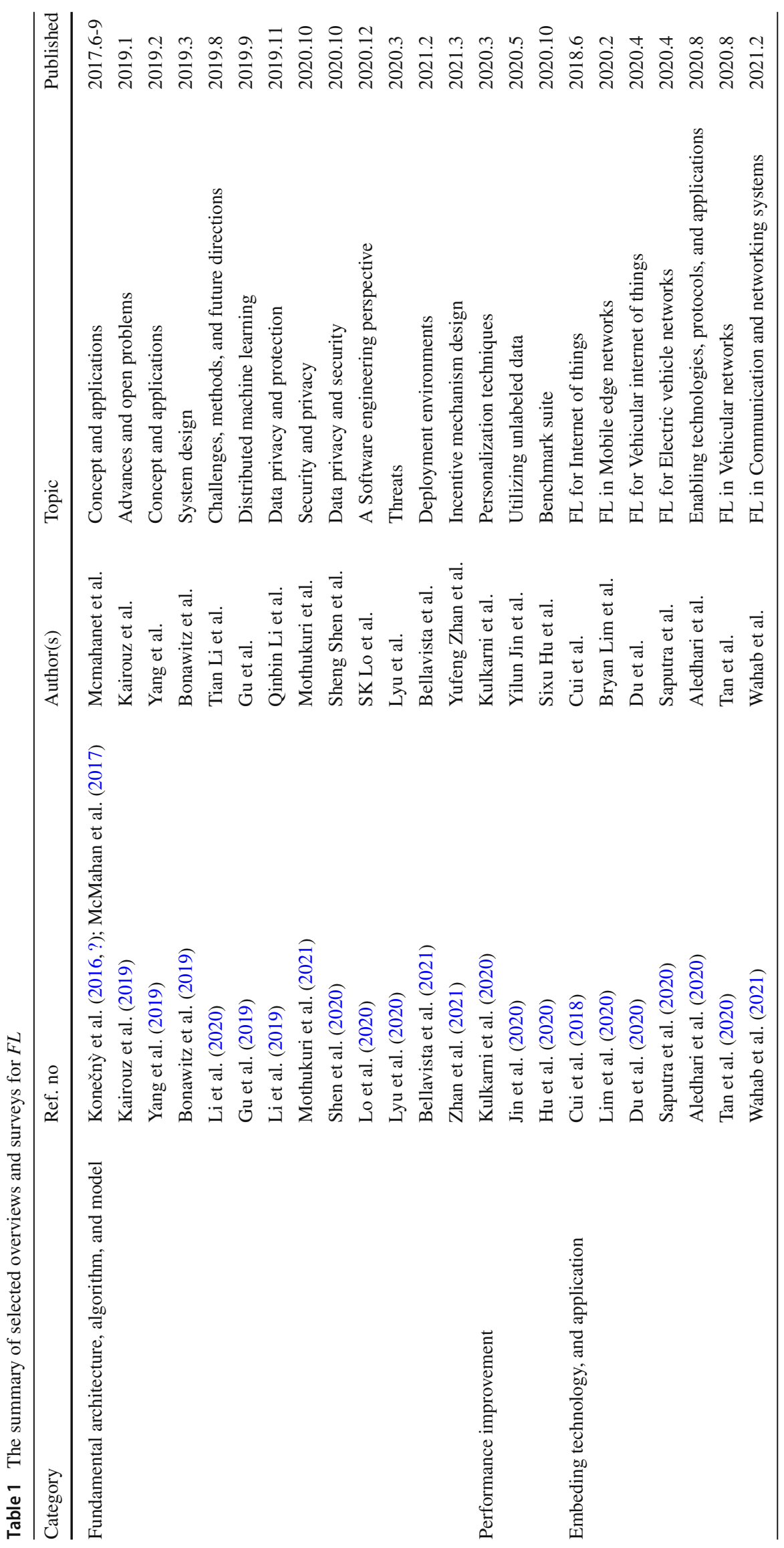




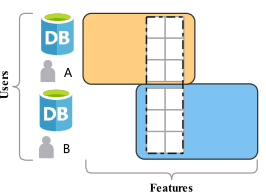

(a) Horizontal Federated Learning

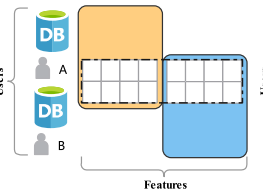

(b) Vertical Federated Learning

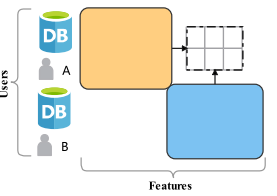

(c) Federated Transfer Learning
Fig. 3 The category of data partition for $F L$

- Step 3. Central Polymerization. After receiving the calculation results from multiple data holders, the central server aggregates the calculated values. In the aggregation process, efficiency, security, privacy, and other issues need to be considered.

Notably, the work of the $F L$ central server is similar to a distributed machine learning server, which collects the gradients of each data holder and then returns a new gradient after performing aggregation operations in the server.

\subsubsection{Applications of $F L$}

Currently, $F L$ has been integrated with other emerging technologies by many scholars to enable industrial applications, such as the efficiency improvement of mobile and wireless communication (Konecný et al. 2016; Sattler et al. 2020; Reisizadeh et al. 2020; Li et al. 2020; Niknam et al. 2020), edge computing (Wang et al. 2019; Doku et al. 2021; Lu et al. 2020; Fantacci and Picano 2020; Wang et al. 2019; Li et al. 2020; Lim et al. 2020), health care (Rieke et al. 2020; Bogdanova et al. 2020; Zerka et al. 2020), Internet of Things (Savazzi et al. 2020; Yang et al. 2020; Yuan et al. 2020; Qolomany et al. 2020; Briggs et al. 2020; Lim et al. 2020; Gao et al. 2020; Kamel and Mougy 2020; Imteaj and Amini 2019), Internet of Vehicles (Samarakoon et al., 2020; Hsu et al., 2020; Du et al., 2020), anomaly detection (Nguyen et al. 2019; Weinger et al. 2020), smart city (Jiang et al. 2020), financial fraud identification (Fan et al. 2020), visual object detection (Liu et al. 2020) and fog computing (Zhou et al. 2020; Cai et al. 2020). It can be seen that $F L$ is prominent in industrial applications for privacy-sensitive data and the processing of non-IID data. Practical industrial-scale applications are not yet sufficient, but theoretical preparations are relatively well established.

\subsubsection{Open-source frameworks of $F L$}

There are currently a few open-source frameworks for researchers and developers to build $F L$ systems. A summary of such frameworks is listed in Tab 3.
Table 2 The summary of acronyms and definitions

\begin{tabular}{ll}
\hline Acronym & Definition \\
\hline$F L$ & Federated learning \\
$H F L$ & Horizontal federated learning \\
$V F L$ & Vertical federated learning \\
$F T L$ & Federated transfer learning \\
$B C F L$ & The integration of \\
& Blockchain and \\
federated learning \\
DDoS & Artificial intelligence \\
$S P O F$ & Distributed denial of service \\
$P o W$ & Single point of failure \\
PoS & Proof of work \\
$D P o W$ & Proof of stake \\
$D P o S$ & Delayed proof-of-work \\
$P B F T$ & Delegated proof-of-stake \\
$d B F T$ & Practical byzantine fault tolerance \\
PooL & Delegated byzantine fault tolerance \\
$I o V$ & Verify the pooling \\
$I o T$ & Internet of vehicles \\
$D T W N$ & Internet of things \\
$5 G$ & Digital twin wireless network \\
$6 G$ & 5th Generation mobile networks \\
\hline & 6th Generation mobile networks \\
\hline &
\end{tabular}

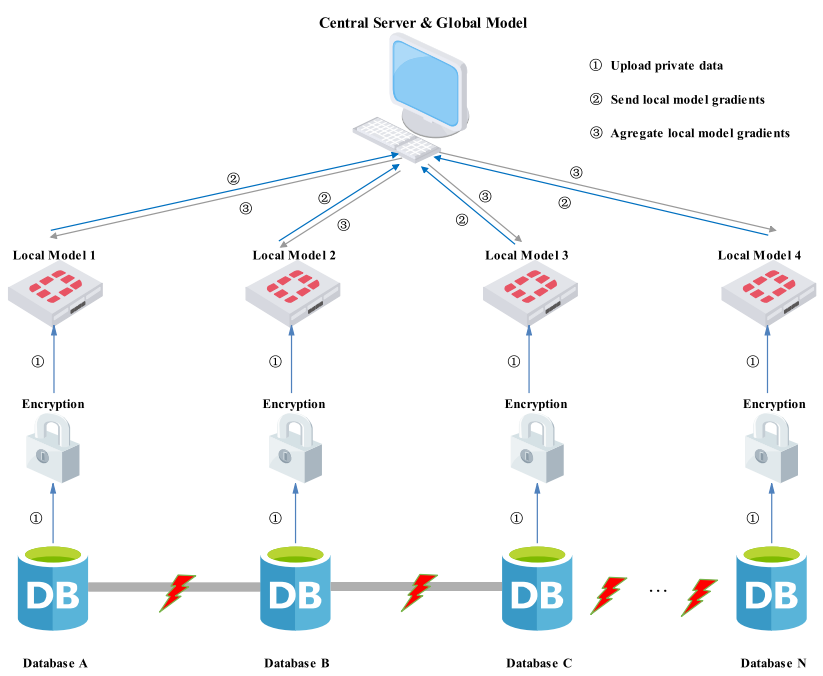

Fig. 4 The workflow of $F L$

\subsection{Blockchain ecosystem}

\subsubsection{Overview of Blockchain}

Blockchain is essentially a decentralized distributed database. All the interactive records (transactions) generated in the system are linked into chains as blocks and stored in each section in time. Furthermore, each transaction is guaranteed by cryp- 
Table 3 The summary of open-source frameworks of $F L$

\begin{tabular}{llllll}
\hline Project & Publisher & Framework & Open source & Refs. & Github \\
\hline $\begin{array}{l}\text { Tensorflow } \\
\text { Federated }\end{array}$ & Google & Tensorflow & Code blocks & XXXX (XXXX) & https://github.com/tensorflow/federated \\
$\begin{array}{l}\text { PySyt } \\
\text { FATE }\end{array}$ & Ryffel et.al & PyTorch & Code blocks & Ryffel et al. (2018) & https://github.com/OpenMined/PySyft \\
PaddleFL & Webank & KubeFATE & API & XXXX (XXXX) & https://github.com/FederatedAI/FATE \\
FedML & Baidu & PaddlePaddle & API & Ma et al. (2019) & https://github.com/PaddlePaddle/PaddleFL \\
& University of & worker-oriented & API & He et al. (2020) & https://github.com/FedML-AI/FedML \\
& Southern & program & & & \\
\hline
\end{tabular}

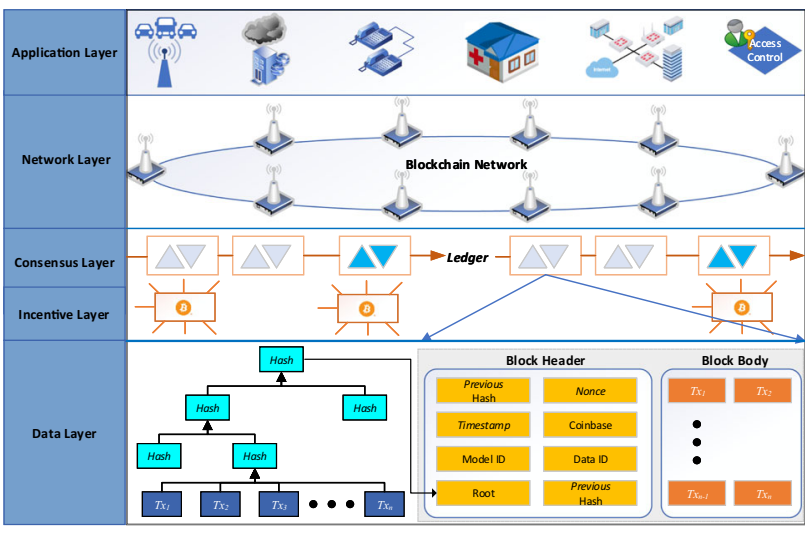

Fig. 5 The architecture of Blockchain

tography and $P o W$ algorithms that cannot be tampered with or forged, so each node in the system can achieve secure peer-to-peer transactions. As Fig. 5 shows, a block consists of a block header containing metadata and some transaction records. These blocks are linked by the hash pointer of the block header to form a complete ledger, which is the narrow definition of Blockchain. More precisely, from the bottom to the top, the Blockchain is composed of the data layer, incentive mechanism, consensus layer, network layer, and application layer (Zheng et al. 2017; Fan et al. 2021; Zheng et al. 2018; Lu 2018; Liang et al. 2019).

Based on different application scenarios and designed systems, the Blockchain is generally divided into public chain, consortium chain, and private chain. Table 4 presents the comparison of three different types of Blockchain. Generally, different types of Blockchain are selected according to the requirements of different business scenarios (Liang et al. 2021). However, in a broad sense, only the public chain can meet the original design intention of the Blockchain.

\subsubsection{Consensus mechanism}

The most fundamental consensus mechanism of Blockchain is the proof-of-work $(P O W)$. A node chooses to store the hash value of a specific block in the current block and then mines it. Once successfully linked, it means that the node accepts the transactions of this block and all previous blocks linked by this block. In addition to $P o W$, there are many other types of consensus mechanisms. Table 5 lists several typical consensus mechanisms and gives a comparative explanation.

\subsubsection{Smart contract}

The smart contract can digitally verify the negotiated or executed contracts and allow trusted transactions without a third party. Besides, these transactions are traceable, and irreversible (Huang et al. 2019). Thus, the success of Ethereum has contributed to the realization of smart contracts. As shown in Fig. 6, it includes transaction processing and preservation mechanism and a complete state machine for accepting and processing various smart contracts. Smart contracts bring great versatility and adaptability to the Blockchain. It is because of the smart contract functionality that various algorithms, including $F L$, can be deployed on the Blockchain.

\section{Structure design of $B C F L$}

This section outlines the main characteristics of the Blockch ain-based federated learning $(B C F L)$ framework. More precisely, in Sect. 4.1, we first introduce the $B C F L$ architecture arising from the integration of Blockchain and $F L$. Then, we present the design of data storage and the deployed platform of $B C F L$ in Sects. 4.2 and 4.3, respectively.

\subsection{Architecture of BCFL}

The first related research focused on the construction of $B C F L$ has been proposed by Kim et al. (2018). The main concept underlying the $B C F L$ is to solve the issues on private exchange and reward mechanisms by using Blockchain (Hieu et al. 2020). Subsequent related studies, such as Mugunthan et al. (2020), Kang et al. (2020), Ma et al. (2020), and Majeed and Hong (2019), have also built some contributions on this foundation, but only introducing some small-scale improve- 
ments. Besides, to make an intuitive display, a demo of $B C F L$ has been proposed by Zhang et al. (2020). However, these follow-up studies all adopted this basic design structure, as shown in Fig. 7.

Specifically, the Blockchain mainly serves as a central database for the $F L$ system, which is fully decentralized and privacy-protected. Therefore, the main goal is to reward the clients according to the quality of their contributions while
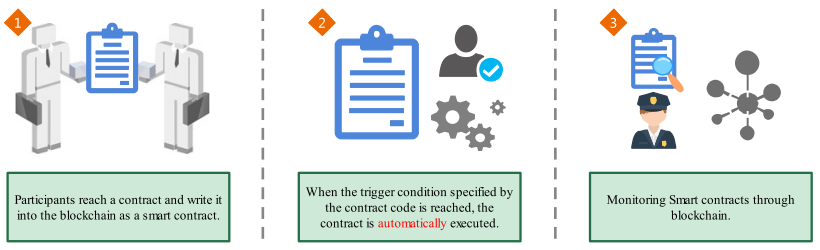

Fig. 6 Smart contract
Table 4 Taxonomy of Blockchain systems

Table 5 The summary of Consensus in Blockchain

\begin{tabular}{llll}
\hline Blockchain & Participants & Characteristics & TPS \\
\hline Public & Anyone & Decentralized & 3-20 data writes per second \\
Consortium & Authorized nodes & Partially centralized & 1000 data writes per second \\
Private & Authorized nodes & Centralized & 1000 data writes per second \\
\hline
\end{tabular}

\begin{tabular}{|c|c|c|}
\hline Consensus & Merits & Weakness \\
\hline \multirow[t]{3}{*}{ PoW } & $\begin{array}{l}\text { Complete centralization, nodes } \\
\text { free access }\end{array}$ & $\begin{array}{l}\text { Waste of energy and difficult to } \\
\text { reduce the confirmation time of } \\
\text { blocks }\end{array}$ \\
\hline & Simple algorithm & $\begin{array}{l}\text { Prone to forking and need to wait } \\
\text { for multiple forks to reach } \\
\text { consistency }\end{array}$ \\
\hline & $\begin{array}{l}\text { The cost of destruction is } \\
\text { huge(destroyer exceed } 50 \% \text { ) }\end{array}$ & \\
\hline \multirow[t]{2}{*}{$\operatorname{PoS}$} & $\begin{array}{l}\text { cLow performance requirements } \\
\text { for nodes }\end{array}$ & $\begin{array}{l}\text { No final consistency, need } \\
\text { checkpoint mechanism to } \\
\text { compensate and finality }\end{array}$ \\
\hline & Short consensus time & \\
\hline DPoW & $\begin{array}{l}\text { Significantly reduce the number of } \\
\text { nodes involved in validation }\end{array}$ & $\begin{array}{l}\text { Sacrifices the concept of } \\
\text { decentralization, not suitable for } \\
\text { public chains }\end{array}$ \\
\hline \multirow[t]{2}{*}{$D P o S$} & Energy conservation & $\begin{array}{l}\text { Slightly more centralized, e.g., } \\
\text { participants with high equity can } \\
\text { vote to make themselves a } \\
\text { validator. }\end{array}$ \\
\hline & Rapidity & \\
\hline$P B F T$ & $\begin{array}{l}\text { High consensus efficiency for high } \\
\text { frequency trading }\end{array}$ & $\begin{array}{l}\text { The existence of cryptocurrency } \\
\text { and the incentive mechanism will } \\
\text { create a Matthew effect making } \\
\text { the poor poorer and the rich } \\
\text { richer in the community } \\
\text { The system will stop when only } \\
33 \% \text { of the nodes are left running }\end{array}$ \\
\hline$d B F T$ & $\begin{array}{l}\text { Highly fault-tolerant with } \\
\text { bookkeeping done by multiple } \\
\text { nodes }\end{array}$ & $\begin{array}{l}\text { The system will not be able to } \\
\text { provide services when more than } \\
\text { one-third of the bookkeepers stop } \\
\text { working }\end{array}$ \\
\hline
\end{tabular}

Every block has finality

The algorithm has a strict mathematical proof that it will not bifurcate

PooL No cryptocurrency required

Less decentralized

Second-level consensus verification 


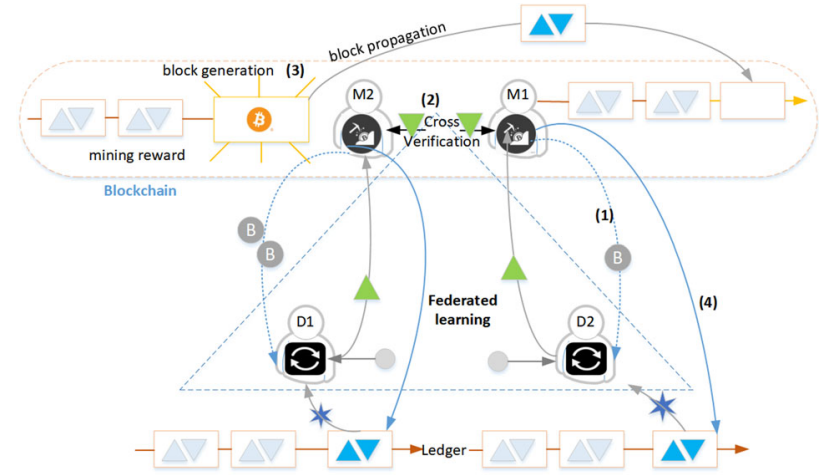

Fig. 7 The architecture of $B C F L$

protecting the privacy of the underlying dataset and fending off malicious adversaries.

\subsection{Data storage}

As with any distributed system, $F L$ bears the privacy leakage challenge. For $B C F L$, the Blockchain plays a pivotal role in solving this problem (Liang et al. 2020). Indeed, the decentralized functioning of Blockchain enables to make $F L$ fault-tolerant (Shayan et al. 2021), and can help to avoid attacks effectively. More precisely, to better solve the security problem of data storage, many studies try to make further improvements based on ordinary Blockchain. For example, a new ring decentralization algorithm (Hu et al. 2020), and an innovative committee consensus mechanism (Li et al. 2021) was shown to be feasible solutions for improving decentralized FL performance and reducing consensus computation, respectively. In summary, the Blockchain data storage model can protect the privacy of a single client update and maintain the large-scale performance of the global model.

\subsection{Deployed platform}

In $B C F L$, the functions of the Blockchain layer need to be implemented with the support of a platform. Different Blockchain platforms have different characteristics. For example, public chains provide stable performance, consortium chains provide robust security, and private chains provide more customization features. From a careful analysis of the literature, the current $B C F L$ mainly adopts four platforms: Ethereum, Hyperledger Fabric, EOS, and Custom Blockchain. The features comparison of these platforms is shown in Table 6.

\subsubsection{Ethereum}

As the earliest programmable Blockchain, Ethereum is Turing-Complete (Buterin XXXX). The work proposed by
Nagar (2019) deploys the BCFL platform using an unlicensed side chain, using a technique proposed by layer 2 extension. Moreover, based on smart contracts in Ethereum, Mugunthan et al. (2020) proposes the BlockFLow architecture, which initially realizes accountable and privacy-preserving $F L$ through a novel contribution scoring procedure. Similarly, Baffle (Ramanan et al. 2020) and ChainFL (Korkmaz et al. 2020) are both Etherium-based FL systems, which use smart contracts to coordinate round partitioning, model aggregation, and update tasks in $F L$.

\subsubsection{Hyperledger Fabric}

As an open-source project, Fabric is initiated by the Linux Foundation and maintained by several corporate organizations. Zhang et al. (2020) demonstrate $F L$ training neural network model on $F L$ client's physical distribution dataset. The underlying communication between the server and the client uses the new Blockchain-based protocol on the secure data exchange system.

\subsubsection{EOS}

The Enterprise Operating System $(E O S)$ is a Blockchainbased operating system designed for commercial distributed applications (Grigg XXXX). For example, an EOS-based $F L$ framework is proposed in Martinez et al. (2019), in which the model owner $O$ has the total liability of payment for the device and producer work, as opposed to devices $D$ needing to pay for their transactions.

\subsubsection{Custom Blockchain}

Although there are many well-established public chains or consortium chains on the market, many researchers still choose to load FL systems with Custom Blockchains. The main reason is that the Custom Blockchain allows better flexibility, programmability, and extensibility. In particular, the work of Kim et al. (2020) proposes BlockFL, an architecture based on a Custom Blockchain in which local learning model updates are exchanged and validated. Similarly, Lu et al. (2020) propose a system consisting of a dual-module containing a permission Blockchain module and a FL module.

\section{Model improvement in BCFL}

$F L$ is essentially a kind of machine learning. Therefore, its learning performance, efficiency, and security are important aspects to take into account. For this reason, several studies have been proposed to make appropriate improvements to the $B C F L$ and enhance the above model performance. Table 
Table 6 The summary of Deployed Platform for $B C F L$

\begin{tabular}{|c|c|c|c|c|c|}
\hline Platform & Blockchain type & Consensus & Identity & Recent studies & Refs. \\
\hline Ethereum & Public & PoW, PoS & Anonymity & $\begin{array}{c}\text { BlockFLow, } \\
\text { BAFFLE, } \\
\text { Chain FL }\end{array}$ & $\begin{array}{l}\text { Nagar (2019), } \\
\text { Mugunthan et al. } \\
\text { (2020), Ramanan } \\
\text { et al. (2020), } \\
\text { Korkmaz et al. } \\
\text { (2020) }\end{array}$ \\
\hline $\begin{array}{l}\text { Hyperledger } \\
\text { Fabric }\end{array}$ & Consortium & SOLO, Kafka & Known identity & DEMO & Zhang et al. (2020) \\
\hline EOS & Consortium & DPoS,BFT & Known identity & BlockFL & $\begin{array}{l}\text { Martinez et al. } \\
\text { (2019) }\end{array}$ \\
\hline $\begin{array}{l}\text { Custom } \\
\text { Blockchain }\end{array}$ & Private & PBFT & Known identity & $\begin{array}{l}\text { BlockFL*, } \\
\text { Secure- } \\
\text { DataShar- } \\
\text { ing }\end{array}$ & $\begin{array}{l}\text { Kim et al. (2020), Lu } \\
\text { et al. (2020) }\end{array}$ \\
\hline
\end{tabular}

7 summarizes the current effective attempts to improve the BCFL.

\subsection{Performance improvement}

$F L$ is a distributed machine learning method that supports local storage of data. In this method, the client implements training through interactive gradient values. Therefore, the underlying idea for improving the accuracy of the model is similar to classical machine learning.

\subsubsection{Fault tolerant enhancement}

ChainFL proposed in Korkmaz et al. (2020) achieves encouraging results on the Modified National Institute of Standards and Technology database digit recognition task (MNIST) and Canadian Institute for Advanced Research image classification task (CIFAR-10). Such results demonstrate that the $B C F L$ model can enhance the system fault tolerance without losing the corresponding model performance compared to the traditional FL model.

\subsubsection{Solving non-IID issues}

The ID labels of data samples have a significant impact on the accuracy of machine learning models. To address the problem that user-generated data samples across devices are likely to become non-IID, Jeong et al. (2018) proposed federated augmentation( $F A u g$ ), a data augmentation scheme that collectively trains generative models on each device to enhance the local data to generate IID datasets.

\subsection{Efficiency tracking and improvement}

For industrial areas such as languages and games, large-scale computations still have high demands on overall algorithm performance (Ogiela and Ogiela 2009). Thus, the tracking and measurement of the algorithm's efficiency are therefore crucial.

\subsubsection{Replace oracle service with chaincode}

The efficiency of the database will have an appreciable impact on the efficiency of FL. Again, the smart contract function in the Blockchain can replace the oracle service to achieve the data access function. The work of Drungilas et al. (2021) uses chaincode in Hyperledger structures instead of oracle services in the database and compares the runtime of functions executed using either chaincode or oracle services, demonstrating that negligible differences between implementations justify the flexible choice of model.

\subsubsection{Setting weight parameter}

Blockchain allows the performance of algorithms to be securely stored and recorded, and in particular, the long-term trend of $F L$ can be tracked, depicting the overall situation and future dynamics of algorithm efficiency during operation. Therefore, weights based on each client's local learning accuracy and weights based on each client's frequency of participation can be used to achieve higher stability and faster convergence times to target accuracy. For instance, Kim and Hong (2019) propose a local learning weighting method for node recognition. This method selects nodes according to the participation frequency and data and weights to achieve fast convergence and stable learning accuracy. 


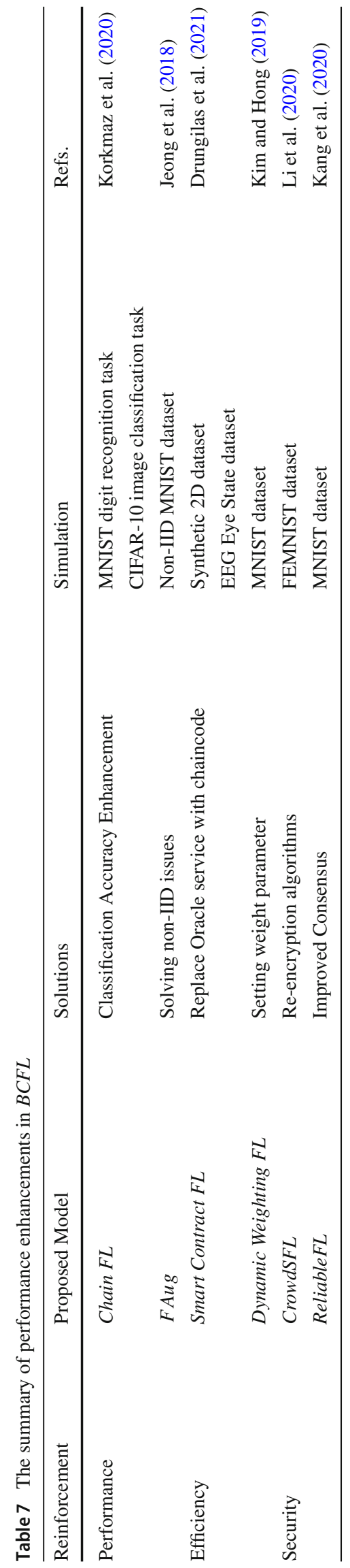

\subsection{Security improvement}

Existing schemes have proven that the Blockchain-based decentralized control mechanism of Blockchain can effectively prevent risks such as SPOF (Liu et al., 2020; Kim and Kim, 2020; Firdaus and Rhee, 2021; Dwivedi et al., 2021; Ruggeri et al., 2020), $D D o S$ attacks (Li et al., 2019; Saad et al., 2019; Rodrigues et al., 2017; Houda et al., 2019; Elisa et al., 2020), and poisoning attacks (Liang et al., 2019; Barański and Konorski, 2020; Rathore et al., 2019). However, the considerable computing power and storage cost of standard solutions are still critical challenges.

\subsubsection{Re-encryption algorithms}

Another possibility to achieve low-cost security improvements is to use re-encryption algorithms (Han et al. 2020). For example, the work by Li et al. (2020) proposes a crowdsourcing framework called CrowdSFL, in which a re-encryption algorithm based on the ElGamal cryptosystem is designed to ensure that interaction values and other information are not exposed to other participants outside the workflow. In this way, users can realize crowdsourcing with less overhead and higher security.

\subsubsection{Improved consensus}

As mentioned in Sect. 4.2, the consensus mechanism in the Blockchain can better ensure the security and privacy of $F L$ 's data storage. Therefore, the appropriate improvement of the consensus mechanism can make $F L$ more suitable for different scenarios and data. A reliable worker selection scheme for $F L$ tasks proposed in Kang et al. (2020) introduces the concept of reputation as a metric to identify trusted and reliable workers in joint to prevent unreliable updates.

\section{Incentive mechanism in BCFL}

$F L$ participants pay for computational resources. However, the training and commercialization of models are not instantaneous, and therefore, there is some delay before the federation reimburses participants. In this section, we outline the incentive mechanism underlying the $B C F L$. More precisely, in Sect. 6.1, we summarize the current attempts to apply Blockchain technology in handling lazy clients, while in Sects. 6.2 and 6.3 we assess the client contribution and compelling motivation, respectively.

\subsection{Handling lazy clients}

Basic $F L$ does not take into account the identification of lazy clients and lacks incentives for influential learning clients. 
Some studies have already begun to try the node incentive of FL, such as Ng et al. (2020), Khan et al. (2020). However, since there is no actual token mechanism design, these studies mainly focus on documentation, detection, and simulation. In contrast, Block-FL's incentive mechanism deals with lazy nodes more practically. Typically, the works of $\mathrm{Li}$ et al. (2020) and Li et al. (2021) propose and evaluate the learning performance of Blade-FL with bounds that are convex concerning the total number of rounds $K$ and optimize the computational resource allocation to minimize the upper bound.

\subsection{Assessing client contribution}

To sustain the long-term engagement of the high-quality data owners (especially enterprises), the $F L$ system needs to provide appropriate incentives based on the accurate evaluation of computational contributions. The systems in $F L$ can be synchronous or asynchronous, depending on whether they use communication or not. In practice, the system functionality of $F L$ can be well realized only if the computational work of the participating nodes is reasonably and well evaluated. The current, reliable methods mainly include a joint learning framework based on Blockchain protocol (Ma et al. 2021) and a new measurement standard based on verification error (Martinez et al. 2019). Similarly, some of these methods introduce the concept of competition to prevent workers from deviating from the protocol (Ogiela et al. 2016), rewarding only those who contribute (Toyoda et al. 2020).

\subsection{Effective motivation}

Based on the contribution score assessment, part of the $B C F L$ model attempts to incentive highly reputable mobile devices with high-quality data to participate in $F L$ (Kang et al. 2019). The peer-to-peer payment system is a natural profit allocation mechanism in the Blockchain. Taking inspiration from that mechanism, the work of Liu et al. (2020) proposes a support vector machine-based profit allocation framework based on the proof of Shapley protocol. On the other hand, the framework proposed in Cai et al. (2020) is based on evaluating the fractions of the dataset for the corresponding share rewards and a framework of reasonable contribution scores generated by both protocols.

\section{Industrial applications of BCFL}

Due to the strong adaptability exhibited by $B C F L$, there is an increasing trend of its wide application. This section mainly studies the industrial applications of BCFL. As shown in Table 8 , we divide these applications into nine areas and summarize the benefits and improvements brought by the corresponding research.

\subsection{Data processing in health care}

The health care industry is in a prominent position in using data to create value and improve human health. However, it has been proved that the traditional methods used to alleviate the privacy problems of health data are insufficient to protect personal interests. For this reason, it is easy to guess that medical data is highly privacy sensitive. $B C F l$ can be an effective solution to mitigate the problems mentioned above since it can perfectly meet the data processing requirements in the field of medical and health care. In particular, $B C F l$ not only completes the modeling requirements of physical therapy data but also avoids privacy leaks on relevant data. For instance, a new agent model based on $B C F L$ is proposed in Dp et al. (2021), as a real-time medical data processing system. Again, to strengthen the privacy of health care data, the model proposed in Passerat-Palmbach et al. (2019) adopts the integration of unique privacy protection technology based on a protocol composed of protected hardware components and the native Ethereum cryptographic toolkit. Finally, the work of Passerat-Palmbach et al. (2020) also uses a similar model, and on this basis, it strengthens the incentive mechanism of data operation.

\subsection{Anomaly detection in network security}

Open networks and service sharing scenarios are complex and varied, leading to serious security challenges ( $\mathrm{Li}$ et al. 2021). In the $F L$ setting, adversaries have more opportunities to poison a local machine learning model with malicious training samples, thus affecting the results of $F L$ and evading detection. However, the work of Preuveneers et al. (2018) shows that audit machine learning models using an anomaly detection algorithm that detects incremental updates recorded on a Blockchain ledger can effectively prevent attacks. For the same purpose, the framework proposed by Desai et al. (2020) uses smart contracts to detect and punish attackers through fines automatically.

\subsection{Device failure and anomaly detection in IoT}

Device fault detection is one of the most critical issues in the industrial Internet of Things (IIoT). However, in traditional $I o T$ device fault detection, client devices need to upload raw data to a central server for model training, which carries the risk of leakage of sensitive business data (Zhao et al. 2021). Given the sensitivity, massive volume, fragmentation, and security of multi-party data computation in IoT environment, the works of Yin et al. (2020), and Rahman et al. (2020) both propose a $B C F L$-based learning approach for device 


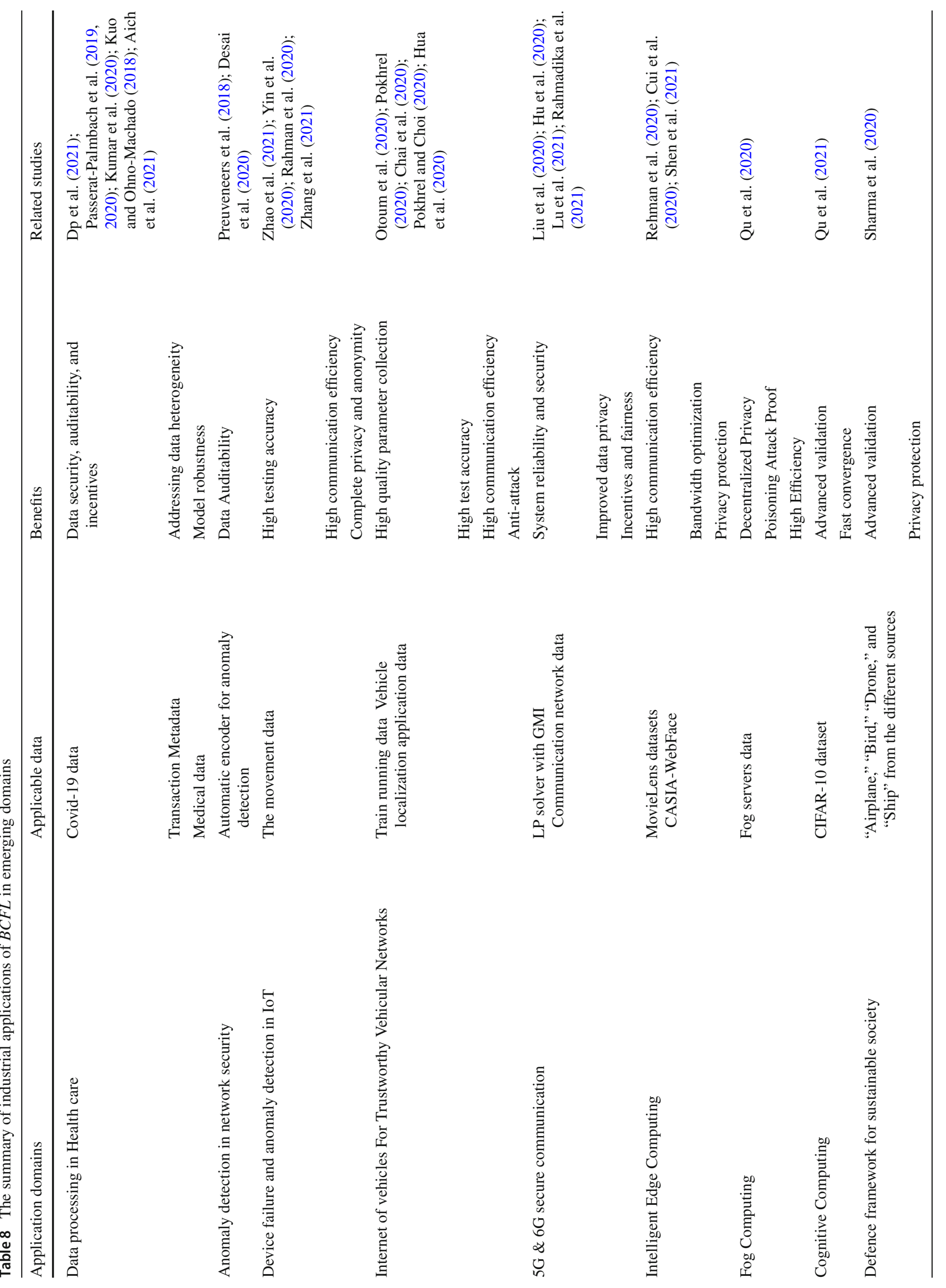


fault detection in IoT. In particular, to solve the data heterogeneity problem in IoT fault detection, Zhang et al. (2021) proposed a novel centroid distance weighted federated averaging (CDW_FedAvg) algorithm. In detail, this algorithm effectively enhances the applicability and model accuracy by taking the distance between positive and negative classes of each client dataset as the basis for calculation.

\subsection{Internet of vehicles for trustworthy vehicular networks}

On the Internet of Vehicles $(I o V)$, sharing data between vehicles for collaborative analysis can improve the driving experience and service quality (Xu et al. 2021). However, efficiency, security, and privacy issues have become obstacles for data providers to participate in the data sharing process (Meng et al. 2021; Pokhrel and Choi 2020; Zou et al. 2021). Fortunately, the $B C F L$ framework is a suitable solution to the contradiction between large-scale data sharing and privacy protection. More precisely, the fundamental applications of $B C F L$ deal with using the validation and consensus mechanisms within the Blockchain to secure IoV data and jointly ensuring trustworthy shared training for mutual machine learning models on decentralized end devices (Otoum et al. 2020). In detail, such operations are carried out by adapting instant block validation at the Blockchain level (Pokhrel 2020) and assessing the trustworthiness of vehicle observations during data collection (Chai et al. 2020). On this basis, the work of Pokhrel and Choi (Pokhrel and Choi 2020) uses the consensus mechanism of Blockchain to manage data without any centralized training or coordination. Meanwhile, the characteristics of controllable networks and $B C F L$ parameters (such as retransmission limit, block size, block arrival rate, and frame size) can better capture their impact on system-level performance. Finally, some researchers have deployed $S V M$ (Hua et al. 2020), and DRL algorithms to improve the efficiency.

\subsection{G and 6G for secure communication}

In recent years, a large number of new applications requiring different network services have emerged. To secure $F L$ in $5 G$ communication, the main current solutions are Blockchain authorization (Liu et al. 2020) and decentralized federated slicing architecture (Hu et al. 2020). Furthermore, the work of Lu et al. (2021) proposed a digital twin wireless network $(D T W N)$ scheme which moved real-time data processing and computing to the edge plane by merging digital twins into wireless networks.

\subsection{Intelligent edge computing}

Edge computing architecture can quickly process the data collected by the Internet of Things (IoT) Zou et al. (2021). Based on the concept of Blockchain reputation perception for fine-grained $F L$, the model proposed in Rehman et al. (2020) can ensure credible collaborative training in mobile edge computing systems. Again, the work of Cui et al. (2020) proposes to apply a compression algorithm of $F L$, assisted by the Blockchain, to predict the content caching of files. On the other hand, as shown in Shen et al. (2021), a new attribute inference attack is proposed. This attack exploits the unexpected attribute leakage of $F L$ aided by Blockchain in intelligent edge computing.

\subsection{Fog computing}

As an extension of cloud computing and the foundation of $I o T$, fog computing is experiencing rapid growth. Indeed, fog computing has the potential to alleviate some thorny issues, such as network congestion, latency, and local autonomy. However, privacy concerns and consequent inefficiencies are slowing down the performance of fog computing (Huang et al. 2019). FL-Block proposed in Qu et al. (2020) modifies the structure of the fog server by storing global updates on the Blockchain to secure the global updates, allowing the end devices to maintain the global model and coordinates based on distributed consensus.

\subsection{Cognitive computing}

Cognitive computing is used to teach a computer to think like a human brain, not just to develop an artificial system. In particular, with the success of AlphaGo and other AI algorithms, cognitive computing has also ushered in a vast development. In this context, the work of Qu et al. (2021) introduces a $B C F L$-based customized reward system to promote public equipment to participate in high-performance industries by deploying Blockchain as the underlying architecture.

\subsection{Sustainable society}

Defense organizations and armed forces are crucial elements for the protection and survival of a nation. However, ensuring these elements requires robust networks and computing power to coordinate intelligence and information processing efficiently. Moreover, given the highly classified nature of national data, Sharma et al. (2020) propose a distributed computational defense framework for a sustainable society using Blockchain technology and $F L$ features. In particular, the proposed framework enables us to infer battlefield states while protecting the privacy of sensitive data. 


\section{Conclusions and future research directions}

This paper presents a survey on the applicability and integration of Blockchain with federated learning $F L$. More precisely, we denote this integration as the Blockchain-based federated learning $(B C F L)$ framework and provide a comprehensive survey of issues related to $B C F L$ implementation. In this paper, we first provide a basic description of the definitions and ecosystems characterizing Blockchain and $F L$. Then, we present the structure design of $B C F L$ as a whole and summarize the feasible deployment platforms. Next, we discuss the model improvement of $F L$ through the introduction of Blockchain. After that, we survey the research related to Blockchain incentives as an element to improve $F L$ systems. Finally, we summarize the full range of possible applications of $B C F L$ in the industry.

In conclusion, the combination of Blockchain and $F L$ is an auspicious research direction, as it can better ensure data security and privacy in the case of abundant data. In addition, this combination makes it possible for more application scenarios to adopt this distributed learning model that does not need to share raw data for joint modeling.

This survey aims to provide a clear view on this topic to ensure that more and more researchers would start working on it. Future research directions could deepen and develop the following aspects:

(1) This paper does not use a cross-referencing and quantitative measure to quantify the overall trends in relevant research. Therefore, future research could consider introducing these elements as a supplement.

(2) Future studies may consider summarizing and classifying the related works from a broader range of perspectives to uncover additional research information relevant to $B C F L$.

(3) $B C F L$ may be applied to increasingly more industrial fields. Consequently, some research efforts may consider more application effects in different industrial fields and make more comparative studies.

Funding This research is supported by the National Natural Science Foundation of China under Grants 61873160 and 61672338 and the Natural Science Foundation of Shanghai under Grant 21ZR1426500.

\section{Declarations}

Conflict of interest The authors declare that they have no known competing financial interests or personal relationships that could have appeared to influence the work reported in this paper.

\section{References}

Aich S, Sinai NK, Kumar S, Ali M, Choi R, Joo MI, Kim HC (2021) Protecting personal healthcare record using blockchain and federated learning technologies. In: 202123 rd International conference on advanced communication technology (ICACT), pp. 109-112

Aledhari M, Razzak R, Parizi RM, Saeed F (2020) Federated learning: a survey on enabling technologies, protocols, and applications. IEEE Access 8:699-725

Barański S, Konorski J (2020) Mitigation of fake data content poisoning attacks in ndn via blockchain. In: 2020 30th International telecommunication networks and applications conference (ITNAC), pp. $1-6$

Bellavista P, Foschini L, Mora A (2021) Decentralised learning in federated deployment environments: a system-level survey. ACM Comput Surveys (CSUR) 54(1):1-38

Bogdanova A, Attoh-Okine N, Sakurai T (2020) Risk and advantages of federated learning for health care data collaboration. ASCEASME J Risk Uncertain Eng Syst, Part A: Civil Eng 6:04020031

Bonawitz K, Eichner H, Grieskamp W, Huba D, Ingerman A, Ivanov V, Kiddon C, Konečnỳ J, Mazzocchi S, McMahan HB et al. (2019) Towards federated learning at scale: system design. arXiv preprint arXiv: 1902.01046

Briggs C, Fan Z, András P (2020) A review of privacy preserving federated learning for private iot analytics. ArXiv, https://arxiv.org/abs/ 2004.11794

Buterin V "Ethereum/wiki, github. [online]. available:," https://github. com/ethereum/wiki

Cai L, Lin D, Zhang J, Yu S (2020) Dynamic sample selection for federated learning with heterogeneous data in fog computing. In: ICC 2020-2020 IEEE International conference on communications (ICC), pp. 1-6

Cai H, Rueckert D, Passerat-Palmbach J (2020) 2CP: decentralized protocols to transparently evaluate contributivity in blockchain federated learning environments. ArXiv, https://arxiv.org/abs/2011. 07516

Chai H, Leng S, Chen Y, Zhang K (2020) A hierarchical blockchainenabled federated learning algorithm for knowledge sharing in internet of vehicles. IEEE Trans Intell Transport Syst. https://doi. org/10.1109/TITS.2020.3002712

Cui L-Z, Su, Ming Z, Chen Z, Yang S, Zhou Y, Xiao W (2020) Creat: Blockchain-assisted compression algorithm of federated learning for content caching in edge computing. In: IEEE Internet Things J, pp. 1-1

Cui L, Yang S, Chen F, Ming Z, Lu N, Qin J (2018) A survey on application of machine learning for internet of things. Int J Mach Learn Cybern 9(8):1399-1417

Desai H, Ozdayi MS, Kantarcioglu M (2020) BlockFLA: Accountable federated learning via hybrid blockchain architecture. In: Proceedings of the eleventh ACM conference on data and application security and privacy

Doku R, Rawat D (2021) Mitigating data poisoning attacks on a federated learning-edge computing network. In: 2021 IEEE 18th Annual consumer communications and networking conference (CCNC), pp. 1-6, 2021

Dp A, Gsb C, Ky D (2021) Agent architecture of an intelligent medical system based on federated learning and blockchain technology. J Inf Secur Appl 58(11):102748

Drungilas V, Vaiciukynas E, Jurgelaitis M, Butkien R, Ceponiene L (2021) Towards blockchain-based federated machine learning: smart contract for model inference. Appl Sci 11(3):1010

Du Z, Wu C, Yoshinaga T, Yau K-LA, Ji Y, Li J (2020) Federated learning for vehicular internet of things: recent advances and open issues. IEEE Open J Comput Soc 1:45-61 
Du Z, Wu C, Yoshinaga T, Yau KLA, Ji Y, Li J (2020) Federated learning for vehicular internet of things: recent advances and open issues. IEEE Open J Comput Soc 1:45-61

Dwivedi SK, Roy P, Karda C, Agrawal S, Amin R (2021) Blockchainbased internet of things and industrial IoT: a comprehensive survey. Secur Commun Netw 2021:1-7

Elisa N, Yang L, Chao F, Cao Y (2020) A framework of blockchainbased secure and privacy-preserving e-government system. In: Wireless networks, pp. 1-11

Fan Y, Zhao G, Lei X, Liang W, Li K, Choo K-K, Zhu C (2021) SBBS: A secure blockchain-based scheme for IoT data credibility in fog environment. IEEE Internet Things J 8(11):9268-9277

Fantacci R, Picano B (2020) Federated learning framework for mobile edge computing networks. CAAI Trans Intell Technol 5:15-21

Fan S, Xu H, Fu S, Xu M (2020) Smart Ponzi scheme detection using federated learning. In: 2020 IEEE 22nd International conference on high performance computing and communications; IEEE 18th International conference on smart city; IEEE 6th International conference on data science and systems (HPCC/SmartCity/DSS), pp. $881-888$

Firdaus M, Rhee K (2021) On blockchain-enhanced secure data storage and sharing in vehicular edge computing networks. Appl Sci 11:414

Gao Y, Kim M, Abuadbba S, Kim Y, Thapa C, Kim K et al. (2020) End-to-end evaluation of federated learning and split learning for internet of things. In: 2020 International symposium on reliable distributed systems (SRDS), pp. 91-100

Google, "Introducing tensorflow federated. [online]. available:," https:// www.tensorflow.org/federated/federated_learning

Gramoli V (2020) From blockchain consensus back to byzantine consensus. Future Gener Comput Syst 107:760-769

Grigg I "Eos-an introduction. [online]. available:," https://eos.io/

Gu R, Yang S, Wu F (2019) Distributed machine learning on mobile devices: a survey. arXiv preprint arXiv: 1909.08329

Han D, Pan N, Li K-C (2020) A traceable and revocable ciphertextpolicy attribute-based encryption scheme based on privacy protection. IEEE Trans Depend Secure Comput. https://doi.org/10.1109/ TDSC.2020.2977646

He C, Li S, So J, Zhang M, Wang H, Wang X, Vepakomma P, Singh A, Qiu H, Shen L, Zhao P, Kang Y, Liu Y, Raskar, Yang Q, Annavaram M, Avestimehr S (2020) FedML: a research library and benchmark for federated machine learning. ArXiv, https://arxiv.org/abs/2007. 13518

Hewa T, Ylianttila M, Liyanage M (2021) Survey on blockchain based smart contracts: applications, opportunities and challenges. J Netw Comput Appl 177:102857

Hieu NQ, Anh TT, Luong NC, Niyato D, Kim D, Elmroth E (2020) Resource management for blockchain-enabled federated learning: a deep reinforcement learning approach. ArXiv, https://arxiv.org/ abs/2004.04104

Houda ZAE, Hafid AS, Khoukhi L (2019) Cochain-SC: an intra- and inter-domain DDoS mitigation scheme based on blockchain using SDN and smart contract. IEEE Access 7:893-907

Hsu YL, Liu C, Samarakoon S, Wei HY, Bennis M (2020) Age-optimal power allocation in industrial IoT: a risk-sensitive federated learning approach. ArXiv, https://arxiv.org/abs/2012.06860

Hu Q, Wang W, Bai X, Jin S, Jiang T (2020) Blockchain enabled federated slicing for $5 \mathrm{G}$ networks with $\mathrm{AI}$ accelerated optimization. IEEE Netw 34:46-52

Hua G, Zhu L, Wu J, Shen C, Zhou L, Lin Q (2020) Blockchain-based federated learning for intelligent control in heavy haul railway. IEEE Access 8:830-839

Huang H, Li K-C, Chen X (2019) Blockchain-based fair three-party contract signing protocol for fog computing. Concurr Comput: Pract Exp 31(22):e4469
Hu S, Li Y, Liu X, Li Q, Wu Z, He B (2020) The oarf benchmark suite: characterization and implications for federated learning systems. arXiv preprint arXiv:2006.07856

Hu Y, Xia W, Xiao J, Wu C (2020) GFL: a decentralized federated learning framework based on blockchain. ArXiv, https://arxiv.org/ abs/2010.10996

Imteaj A, Amini MH (2019) Distributed sensing using smart end-user devices: Pathway to federated learning for autonomous IoT. In: 2019 International conference on computational science and computational intelligence (CSCI), pp. 1156-1161

Jeong E, Oh S, Kim H, Park J, Bennis M, Kim SL (2018) Communication-efficient on-device machine learning: federated distillation and augmentation under non-iid private data. ArXiv, https://arxiv.org/abs/1811.11479

Jiang J, Kantarci B, Oktug S, Soyata T (2020) Federated learning in smart city sensing: challenges and opportunities. Sensors 20:6230

Jin Y, Wei X, Liu Y, Yang Q (2020) Towards utilizing unlabeled data in federated learning: a survey and prospective. arXiv e-prints, pp. arXiv-2002

Kairouz P, McMahan HB, Avent B, Bellet A, Bennis M, Bhagoji AN, Bonawitz K, Charles Z, Cormode G, Cummings R et al. (2019) Advances and open problems in federated learning. arXiv preprint arXiv: 1912.04977

Kamel RM, Mougy AHE (2020) Retrospective sensing based on federated learning in the IoT. In: 2020 IEEE 45th LCN symposium on emerging topics in networking (LCN symposium), pp. 150-161

Kang J, Xiong Z, Niyato D, Xie S, Zhang J (2019) Incentive mechanism for reliable federated learning: a joint optimization approach to combining reputation and contract theory. IEEE Internet Things $\mathbf{J}$ 6:700-714

Kang J, Xiong Z, Niyato D, Zou Y, Zhang Y, Guizani M (2020) Reliable federated learning for mobile networks. IEEE Wirel Commun 27:72-80

Kang J, Xiong Z, Jiang C, Liu Y, Guo S, Zhang Y, Niyato D, Leung C, Miao C (2020) Scalable and communication-efficient decentralized federated edge learning with multi-blockchain framework. ArXiv, https://arxiv.org/abs/2008.04743

Khan LU, Tran NH, Pandey SR, Saad W, Han Z, Nguyen MNH, Hong CS (2020) Federated learning for edge networks: resource optimization and incentive mechanism. IEEE Commun Magaz 58:88-93

Kim YJ, Hong C (2019) Blockchain-based node-aware dynamic weighting methods for improving federated learning performance. In: 2019 20th Asia-pacific network operations and management symposium (APNOMS)

Kim H, Park J, Bennis M, Kim S-L (2020) Blockchained on-device federated learning. IEEE Commun Lett 24:1279-1283

Kim A, Kim M (2020) A study on blockchain-based music distribution framework: focusing on copyright protection. In: 2020 International conference on information and communication technology convergence (ICTC), pp. 1921-1925

Kim H, Park J, Bennis M, Kim SL (2018) On-device federated learning via blockchain and its latency analysis. ArXiv, https://arxiv.org/ abs/1808.03949

Konečnỳ J, McMahan HB, Ramage D, Richtárik P (2016) Federated optimization: Distributed machine learning for on-device intelligence. arXiv preprint arXiv:1610.02527

Konečnỳ J, McMahan HB, Yu FX, Richtárik P, Suresh AT,Bacon D (2016)Federated learning: strategies for improving communication efficiency. arXiv preprint arXiv: 1610.05492

Konecný J, McMahan HB, Yu F, Richtárik P, Suresh AT, Bacon D (2016) Federated learning: strategies for improving communication efficiency," ArXiv, https://arxiv.org/abs/1610.05492

Korkmaz C, Kocas HE, Uysal A, Masry A, Ozkasap O, Akgun B (2020) Chain FL: Decentralized federated machine learning 
via blockchain. In: 2020 Second international conference on blockchain computing and applications (BCCA), pp. 140-146

Kulkarni V, Kulkarni M, Pant A (2020) Survey of personalization techniques for federated learning. In: 2020 Fourth world conference on smart trends in systems, security and sustainability (WorldS4). IEEE pp. 794-797

Kumar S, Jaiswal S (2019) Blockchain: overview, practical implementation \& its uses. Int J Res 6:946-963

Kumar R, Khan A, Zhang S, Wang W, Abuidris Y, Amin W, Kumar J (2020) Blockchain-federated-learning and deep learning models for COVID-19 detection using CT imaging. ArXiv, https://arxiv. org/abs/2007.06537

Kuo TT, Ohno-Machado L (2018) Modelchain: Decentralized privacypreserving healthcare predictive modeling framework on private blockchain networks. ArXiv, https://arxiv.org/abs/1802.01746

Li M, Weng J, Yang A, Lu W, Zhang Y, Hou L, Liu J, Xiang Y, Deng RH (2019) Crowdbc: a blockchain-based decentralized framework for crowdsourcing. IEEE Trans Parallel Distrib Syst 30:1251-1266

Li Z, Jia-n L, Hao J, Wang H, Xian M (2020) Crowdsfl: a secure crowd computing framework based on blockchain and federated learning. Electronics 9:773

Li T, Sahu AK, Talwalkar A, Smith V (2020) Federated learning: challenges, methods, and future directions. IEEE Signal Process Magaz 37(3):50-60

Li X, Jiang P, Chen T, Luo X, Qiaoyan W (2020) A survey on the security of blockchain systems. Future Gener Comput Syst 107:841-853

Li M, Han D, Yin X, Liu H, Li D (2021) Design and implementation of an anomaly network traffic detection model integrating temporal and spatial features. Secur Commun Netw 2021:1-15

Li Y, Chen C, Liu N, Huang H, Zheng Z, Yan Q (2021) A blockchainbased decentralized federated learning framework with committee consensus. IEEE Netw 35:234-241

Liang G, Weller SR, Luo F, Zhao J, Dong ZY (2019) Distributed blockchain-based data protection framework for modern power systems against cyber attacks. IEEE Trans Smart Grid 10:31623173

Liang W, Tang M, Long J, Peng X, Xu J, Li K-C (2019) A secure fabric blockchain-based data transmission technique for industrial internet-of-things. IEEE Trans Ind Inf 15(6):3582-3592

Liang W, Li K-C, Long J, Kui X, Zomaya A (2019) An industrial network intrusion detection algorithm based on multifeature data clustering optimization model. IEEE Trans Ind Inf 16(3):20632071

Liang W, Huang W, Long J, Zhang K, Li K-C, Zhang D (2020) Deep reinforcement learning for resource protection and real-time detection in iot environment. IEEE Internet Things J 7(7):6392-6401

Liang W, Fan Y, Li K-C, Zhang D, Gaudiot J-L (2020) Secure data storage and recovery in industrial blockchain network environments. IEEE Trans Ind Inf 16(10):6543-6552

Liang W, Xie S, Zhang D, Li X, Li K-C (2021) A mutual security authentication method for RFID-PUF circuit based on deep learning. ACM Trans Internet Technol 2:1-20

Liang W, Li Y, Xu J, Qin Z, Li KC Qos prediction and adversarial attack protection for distributed services under dlaas. In: IEEE Transactions on Computers

Liang W, Xiao L, Zhang K, Tang M, He D, Li K-C (2021) Data fusion approach for collaborative anomaly intrusion detection in blockchain-based systems. IEEE Internet Things J. https://doi.org/ 10.1109/JIOT.2021.3053842

Liang W, Zhang D, Lei X, Tang M, Li K-C, Zomaya A (2020) Circuit copyright blockchain: blockchain-based homomorphic encryption for IP circuit protection. IEEE Trans Emerg Topics Comput. https://doi.org/10.1109/TETC.2020.2993032

Lim WYB, Luong NC, Hoang DT, Jiao Y, Liang Y-C, Yang Q, Niyato D, Miao C (2020) Federated learning in mobile edge networks: a comprehensive survey. IEEE Commun Surveys Tutor 22(3):20312063

Lim WYB, Luong NC, Hoang DT, Jiao Y, Liang Y-C, Yang Q, Niyato D, Miao C (2020) Federated learning in mobile edge networks: a comprehensive survey. IEEE Commun Surveys Tutor 22:20312063

Lim HK, Kim JB, Heo JS, Han YH (2020) Federated reinforcement learning for training control policies on multiple IoT devices. Sensors 20:1359

Li S, Qi Q, Wang J, Sun H, Li Y, Yu F (2020) GGS: general gradient sparsification for federated learning in edge computing*. In: ICC 2020 - 2020 IEEE International conference on communications (ICC), pp. 1-7, 2020

Li J, Shao Y, Ding M, Ma C, Wei K, Han Z, Poor H (2020) Blockchain assisted decentralized federated learning (BLADE-FL) with lazy clients. ArXiv, https://arxiv.org/abs/2012.02044

Li J, Shao Y, Wei K, Ding M, Ma C, Shi L,Han Z, Poor H (2021) Blockchain assisted decentralized federated learning (BLADEFL): performance analysis and resource allocation. ArXiv, https:// arxiv.org/abs/2101.06905

Li A, Sun J, Wang B, Duan L, Li S, Chen Y, Li H (2020) LotteryFL: Personalized and communication-efficient federated learning with lottery ticket hypothesis on non-iid datasets. ArXiv, vol. https:// arxiv.org/abs/2008.03371

Liu Y, Peng J, Kang J, Iliyasu AM, Niyato D, El-latif AA (2020) A secure federated learning framework for $5 \mathrm{G}$ networks. IEEE Wirel Commun 27:24-31

Liu Y, Huang A, Luo Y, Huang H, Liu Y, Chen YY, Feng L, Chen T, Yu H, Yang Q (2020) Fedvision: an online visual object detection platform powered by federated learning. In: AAAI

Liu Y, Sun S, Ai Z, Zhang S, Liu Z, Yu H (2020) Fedcoin: a peer-topeer payment system for federated learning. Federated learning. Springer, Cham, pp 125-138

Liu W, Zhang Y, Liu L, Liu S, Zhang H, Fang B (2020) A secure domain name resolution and management architecture based on blockchain. In: 2020 IEEE symposium on computers and communications (ISCC), pp. 1-7

Li Q, Wen Z, Wu Z, Hu S, Wang N, He B (2019) A survey on federated learning systems: vision, hype and reality for data privacy and protection," arXiv preprint arXiv:1907.09693

Lo SK, Lu Q, Wang C, Paik H, Zhu L (2020) A systematic literature review on federated machine learning: from a software engineering perspective. arXiv preprint arXiv:2007.11354

Lu Y (2018) Blockchain: a survey on functions, applications and open issues. J Ind Integr Manag 3(04): 1850015

Lu Y, Huang XH, Dai Y, Maharjan S, Zhang Y (2020) Blockchain and federated learning for privacy-preserved data sharing in industrial IoT. IEEE Trans Ind Inf 16:4177-4186

Lu Y, Huang X, Dai Y, Maharjan S, Zhang Y (2020) Differentially private asynchronous federated learning for mobile edge computing in urban informatics. IEEE Trans Ind Inf 16:2134-2143

Lu Y, Huang X, Zhang K, Maharjan S, Zhang Y (2021) Low-latency federated learning and blockchain for edge association in digital twin empowered 6G networks. IEEE Trans Ind Inf 17:5098-5107

Lyu L, Yu H, Yang Q (2020) Threats to federated learning: a survey. arXiv preprint arXiv:2003.02133

Ma Y, Yu D, Wu T, Wang H (2019) PaddlePaddle: an open-source deep learning platform from industrial practice. Front Data Deomput $1: 105-115$

Ma S, Cao Y, Xiong L (2021) Transparent contribution evaluation for secure federated learning on blockchain. ArXiv, https://arxiv.org/ abs/2101.10572

Majeed U, Hong C (2019) FLchain: Federated learning via mec-enabled blockchain network. In: 201920th Asia-pacific network operations and management symposium (APNOMS), pp. 1-4 
Ma C, Li J, Ding M, Shi L, Wang T, Han Z, Poor H (2020) When federated learning meets blockchain: a new distributed learning paradigm. ArXiv, https://arxiv.org/abs/2009.09338

Martinez I, Francis S, Hafid A (2019) Record and reward federated learning contributions with blockchain. In: 2019 International conference on cyber-enabled distributed computing and knowledge discovery (CyberC), pp. 50-57

McMahan B, Moore E, Ramage D, Hampson S, Arcas BAy (2017) Communication-efficient learning of deep networks from decentralized data. In: Artif Intell Stat PMLR, pp. 1273-1282

Meng X, Xu J, Liang W et al (2021) A lightweight anonymous cross-regional mutual authentication scheme using blockchain technology for internet of vehicles. Comput Electr Eng 95:107431

Mothukuri V, Parizi RM, Pouriyeh S, Huang Y, Dehghantanha A, Srivastava $G$ (2021) A survey on security and privacy of federated learning. Future Gener Comput Syst 115:619-640

Mugunthan V, Rahman R, Kagal L (2020) BlockFLow: an accountable and privacy-preserving solution for federated learning. ArXiv, https://arxiv.org/abs/2007.03856

Nagar A (2019) Privacy-preserving blockchain based federated learning with differential data sharing. ArXiv, https://arxiv.org/abs/1912. 04859

Ng KL, Chen Z, Liu Z, Yu H, Liu Y, Yang Q (2020) A multi-player game for studying federated learning incentive schemes. In: IJCAI

Nguyen TD, Marchal S, Miettinen, Fereidooni H, Asokan N, Sadeghi AR (2019) Dïot: A federated self-learning anomaly detection system for IoT. In: 2019 IEEE 39th International conference on distributed computing systems (ICDCS), pp. 756-767

Niknam S, Dhillon HS, Reed JH (2020) Federated learning for wireless communications: motivation, opportunities, and challenges. IEEE Commun Magaz 58:46-51

Ogiela M, Ogiela U (2009) Secure information splitting using grammar schemes. New challenges in computational collective intelligence. Springer, Berlin, Heidelberg, pp 327-336

Ogiela L, Ogiela M, Ogiela U (2016) Efficiency of strategic data sharing and management protocols. In: 2016 10th International conference on innovative mobile and internet services in ubiquitous computing (IMIS), pp. 198-201

Otoum S, Ridhawi IA, Mouftah H (2020) Blockchain-supported federated learning for trustworthy vehicular networks. In: GLOBECOM 2020-2020 IEEE Global communications conference, pp. 1-6

Passerat-Palmbach J, Farnan T, McCoy M, Harris J, Manion ST, Flannery H, Gleim N (2020) Blockchain-orchestrated machine learning for privacy preserving federated learning in electronic health data. In: IEEE international conference on blockchain (blockchain)

Passerat-Palmbach J, Farnan T, Miller R, Gross M, Flannery H, Gleim B (2019) blockchain-orchestrated federated learning architecture for healthcare consortia. ArXiv, https://arxiv.org/abs/1910.12603

Pokhrel S (2020) WITHDRAWN: towards efficient and reliable federated learning using blockchain for autonomous vehicles. Comput Netw. https://doi.org/10.1016/j.comnet.2020.107431

Pokhrel S, Choi J (2020) Federated learning with blockchain for autonomous vehicles: analysis and design challenges. IEEE Trans Commun 68:4734-4746

Pokhrel S, Choi J (2020) Improving TCP performance over WiFi for internet of vehicles: a federated learning approach. IEEE Trans Vehic Technol 69:6798-6802

Preuveneers D, Rimmer V, Tsingenopoulos I, Spooren J, Joosen W, IlieZudor E (2018) Chained anomaly detection models for federated learning: an intrusion detection case study. Appl Sci 8:2663

Qolomany B, Ahmad K, Al-Fuqaha A, Qadir J (2020) Particle swarm optimized federated learning for industrial IoT and smart city services. In: GLOBECOM 2020-2020 IEEE global communications conference, pp. 1-6
Qu Y, Gao L, Luan TH, Xiang Y, Yu S, Li B, Zheng G (2020) Decentralized privacy using blockchain-enabled federated learning in fog computing. IEEE Internet Things J 7:5171-5183

Qu Y, Pokhrel S, Garg S, Gao L, Xiang Y (2021) A blockchained federated learning framework for cognitive computing in industry 4.0 networks. IEEE Trans Ind Inf 17:2964-2973

Rahmadika S, Firdaus M, Jang S, Rhee K (2021) Blockchain-enabled $5 \mathrm{~g}$ edge networks and beyond: an intelligent cross-silo federated learning approach. Secur Commun Netw 2021:1-14

Rahman M, Hossain MS, Islam MS, Alrajeh N, Muhammad G (2020) Secure and provenance enhanced internet of health things framework: a blockchain managed federated learning approach. IEEE Access 8:71-87

Ramanan P,Nakayama K,Sharma R (2020) Baffle: blockchain based aggregator free federated learning. In: 2020 IEEE International Conference on Blockchain (Blockchain), pp. 72-81

Rathore S, Pan Y, Park JH (2019) Blockdeepnet: a blockchain-based secure deep learning for IoT network. Sustainability 11:3974

Rehman MHU, Salah K, Damiani E, Svetinovic D (2020) Towards blockchain-based reputation-aware federated learning. In: IEEE INFOCOM 2020-IEEE Conference on Computer Communications Workshops (INFOCOM WKSHPS), pp. 183-188

Reisizadeh A, Mokhtari A, Hassani H, Jadbabaie A, Pedarsani R (2020) FedPAQ: A communication-efficient federated learning method with periodic averaging and quantization. ArXivhttps://arxiv.org/ abs/1909.13014

Rieke N, Hancox J, Li W, Milletari F, Roth HR, Albarqouni S, Bakas S, Galtier M, Landman B, Maier-Hein KH, Ourselin S, Sheller MJ, Summers RM, Trask A, Xu D, Baust M, Cardoso MJ (2020) The future of digital health with federated learning. NPJ Digit Med $3: 1-7$

Rodrigues BB, Bocek TM, Lareida A, Hausheer D, Rafati S, Stiller B (2017) A blockchain-based architecture for collaborative DDoS mitigation with smart contracts. FIP International conference on autonomous infrastructure, management and security. Springer, Cham, pp 16-29

Ruggeri A, Celesti A, Fazio M, Galletta A, Villari M (2020) BCBX3DH: a blockchain based improved version of the extended triple diffie-hellman protocol. In: 2020 Second IEEE International Conference on Trust, Privacy and Security in Intelligent Systems and Applications (TPS-ISA), pp. 73-78

Ryffel T, Trask A, Dahl M, Wagner B, Mancuso J, Rueckert D, PasseratPalmbach J (2018) A generic framework for privacy preserving deep learning. ArXiv, https://arxiv.org/abs/1811.04017

Saad M, Spaulding J, Njilla LL, Kamhoua CA, Shetty S, Nyang D, Mohaisen A (2019) Exploring the attack surface of blockchain: a systematic overview. ArXiv, https://arxiv.org/abs/1904.03487

Saleh F (2020) Blockchain without waste: proof-of-stake. Inf Syst Econ eJ

Samarakoon S, Bennis M, Saad W, Debbah M (2020) Distributed federated learning for ultra-reliable low-latency vehicular communications. IEEE Trans Commun 68:1146-1159

Saputra YM, Nguyen DN, Hoang DT, Vu TX, Dutkiewicz E, Chatzinotas S (2020) Federated learning meets contract theory: energyefficient framework for electric vehicle networks. arXiv preprint arXiv:2004.01828

Sattler F, Wiedemann S, Müller K, Samek W (2020) Robust and communication-efficient federated learning from non-iid data. IEEE Trans Neural Netw Learn Syst 31:3400-3413

Savazzi S, Nicoli M, Rampa V (2020) Federated learning with cooperating devices: a consensus approach for massive IoT networks. IEEE Internet Things J 7:4641-4654

Sharma P, Park JH, Cho K (2020) Blockchain and federated learningbased distributed computing defence framework for sustainable society. Sustainable Cities Soc 59:102220 
Shayan M, Fung C, Yoon CJM, Beschastnikh I (2021) Biscotti: a blockchain system for private and secure federated learning. IEEE Trans Parallel Distrib Syst 32:1513-1525

Shen M, Wang H, Zhang B, Zhu L, Xu K, Li Q, Du X (2021) Exploiting unintended property leakage in blockchain-assisted federated learning for intelligent edge computing. IEEE Internet Things $\mathrm{J}$ 8:2265-2275

Shen S, Zhu T, Wu D, Wang W, Zhou W (2020) From distributed machine learning to federated learning: In the view of data privacy and security. Practice and Experience, Concurrency and Computation

Tan K, Bremner D, Le Kernec J, Imran M (2020)F ederated machine learning in vehicular networks: A summary of recent applications. In: 2020 International conference on UK-China emerging technologies (UCET). IEEE, pp. 1-4

Toyoda K, Zhao J, Zhang A, Mathiopoulos P (2020) Blockchainenabled federated learning with mechanism design. IEEE Access 8:744-756

Wahab OA, Mourad A, Otrok H, Taleb T (2021) Federated machine learning: survey, multi-level classification, desirable criteria and future directions in communication and networking systems. IEEE Commun Surveys Tutor 23(2):1342-1397

Wang S, Tuor T, Salonidis T, Leung K, Makaya C, He T, Chan K (2019) Adaptive federated learning in resource constrained edge computing systems. IEEE J Select Areas Commun 37:1205-1221

Wang X, Han Y, Wang C, Zhao Q, Chen X, Chen M (2019) In-edge AI: intelligentizing mobile edge computing, caching and communication by federated learning. IEEE Netw 33:156-165

Webank, "Fate: An industrial grade federated learning framework. [online]. available:," https://fate.fedai.org/

Weinger B, Kim J, Sim A, Nakashima M, Moustafa N, Wu K (2020) Enhancing IoT anomaly detection performance for federated learning. In: 2020 16th International Conference on Mobility, Sensing and Networking (MSN), pp. 206-213

Xiao Y, Zhang N, Lou W, Hou YT (2020) A survey of distributed consensus protocols for blockchain networks. IEEE Commun Surveys Tutor 22:1432-1465

Xiao L, Han D, Meng X, Liang W, Li K-C (2020) A secure framework for data sharing in private blockchain-based WBANs. IEEE Access 8:956-968

Xu G, Li H, Liu S, Yang K, Lin X (2020) Verifynet: secure and verifiable federated learning. IEEE Trans Inf Forens Secur 15:911-926

Xu Z, Liang W, Li K, Xu J, Jin H (2021) A blockchain-based roadside unit-assisted authentication and key agreement protocol for internet of vehicles. J Parallel Distributed Comput 149:29-39

Yang Q, Liu Y, Chen T, Tong Y (2019) Federated machine learning: concept and applications. ACM Trans Intell Syst Technol (TIST) 10(2):1-19

Yang K, Shi Y, Zhou Y, Yang Z, Fu L, Chen W (2020) Federated machine learning for intelligent IoT via reconfigurable intelligent surface. IEEE Netw 34:16-22

Yin B, Yin H, Wu Y, Jiang Z (2020) FDC: A secure federated deep learning mechanism for data collaborations in the internet of things. IEEE Internet Things J 7:6348-6359

Yu L, Duan Y, Li K (2021) A real-world service mashup platform based on data integration, information synthesis, and knowledge fusion. Connect Sci 33(3):463-481

Yuan B, Ge S, Xing W (2020) A federated learning framework for healthcare IoT devices. ArXiv, https://arxiv.org/abs/2005.05083

Zerka F, Barakat S, Walsh SC, Bogowicz M, Leijenaar RTH, Jochems A, Miraglio B, Townend D, Lambin P (2020) Systematic review of privacy-preserving distributed machine learning from federated databases in health care. JCO Clin Cancer Inf 4:184-200

Zhan Y, Zhang J, Hong Z, Wu L, Li P, Guo S (2021) A survey of incentive mechanism design for federated learning. IEEE Trans Emerg Topics Comput 99:1-1
Zhang W, Lu Q, Yu Q, Li Z, Liu Y, Lo SK, Chen S, Xu X, Zhu L (2021) Blockchain-based federated learning for device failure detection in industrial IoT. IEEE Internet Things J 8:5926-5937

Zhang Q, Palacharla P, Sekiya M, Suga J, Katagiri T (2020) Demo: a blockchain based protocol for federated learning. In: 2020 IEEE 28th International conference on network protocols (ICNP), pp. $1-2$

Zhao Y, Zhao J, Jiang L, Tan R, Niyato D, Li Z, Lyu L, Liu Y (2021) Privacy-preserving blockchain-based federated learning for IoT devices. IEEE Internet Things J 8:1817-1829

Zheng Z, Xie S, Dai H, Chen X, Wang H (2018) Blockchain challenges and opportunities: a survey. Int J Web Grid Serv 14:352-375

Zheng Z, Xie S, Dai H, Chen X, Wang H (2017) An overview of blockchain technology: Architecture, consensus, and future trends. In: 2017 IEEE International congress on big data (BigData Congress), pp. 557-564

Zhou Q, Huang H, Zheng Z, Bian J (2020) Solutions to scalability of blockchain: a survey. IEEE Access 8:440-455

Zhou C, Fu A, Yu S, Yang W, Wang H, Zhang Y (2020) Privacypreserving federated learning in fog computing. IEEE Internet Things J 7:782-793

Zou G, Qin Z, Deng S et al (2021) Towards the optimality of service instance selection in mobile edge computing. Knowl-Based Syst 217:106831

Zou Y, Shen F, Yan F, Lin J, Qiu Y (2021) Reputation-based regional federated learning for knowledge trading in blockchain-enhanced IoV In: 2021 IEEE Wireless communications and networking conference (WCNC), pp. 1-6

Publisher's Note Springer Nature remains neutral with regard to jurisdictional claims in published maps and institutional affiliations. 